\title{
Consumo de álcool entre os trabalhadores da saúde da família
}

\section{Alcohol consumption among family health workers}

\author{
Maria Aparecida Carlos \\ Álex Moreira Herval** \\ Liliane Parreira Tannús Gontijo
}

\section{Resumo}

Objetivo: identificar o padrão e a prevalência de consumo de bebida alcoólica entre trabalhadores de equipes de Saúde da Família. Materiais e método: estudo transversal conduzido com 118 trabalhadores da Saúde da Família. Os dados foram coletados por meio um questionário socioeconômico e ocupacional e do Teste de Identificação dos Transtornos do Uso de Álcool, cujos resultados foram analisados de forma descritiva. Resultados: o uso de álcool foi observado em $50 \%$ dos profissionais de saúde. Dos que faziam uso de álcool, $41 \%$ revelaram ter consumido seis ou mais doses de álcool em uma única ocasião (binge), 19\% alegaram ao menos um episódio de binge por mês, $12 \%$ disseram beber mensalmente, $8 \%$ semanalmente e $2 \%$ todos ou quase todos os dias. Conclusões: o uso de álcool entre profissionais da saúde da família foi considerado similar à população brasileira. Contudo, entre os trabalhadores que fazem uso do álcool, chamou a atenção a frequência elevada de uso em binge.

Palavras-chave: Alcoolismo. Atenção primária à saúde. Saúde do trabalhador.

\section{Introdução}

O número de equipes de Saúde da Família está em expansão contínua no Brasil, constituindo-se como um dos principais empregadores de trabalhadores da saúde, entre os quais está o cirurgião-dentista. O trabalho na saúde da família é considerado complexo, aglutinando um conjunto de ações integrais de âmbito individual e coletivo. Nesses serviços, os profissionais devem utilizar saberes e práticas socialmente aceitas, com foco na resolutividade e na atenção integral ${ }^{1}$.

Sendo um trabalho complexo, é necessário estar atento ao fato de que determinadas formas de organização do trabalho podem se tornar fonte de danos à saúde dos trabalhadores, pois atuam como fatores de desgaste que vão além do cansaço físico, abrangendo aspectos físicos e cognitivos ${ }^{2}$.

Estudos científicos sobre a relação entre a organização do trabalho e o uso do álcool ainda são novos. Apesar disso, o álcool é considerado pela classe trabalhadora como um dos principais recursos utilizados para enfrentar as agruras vivenciadas no cotidiano do ambiente laboral, além de ser fonte de prazer e relaxamento ${ }^{3}$. Deve-se considerar, ainda, que o álcool é uma substância licita de fácil acesso e que tem seu consumo frequentemente estimulado pelos meios de comunicação. Além disso, a pressão social para beber, a disponibilidade do álcool, as situações de tensão e o estresse ocupacional são fatores relacionados ao ambiente de trabalho que favorecerem o uso dessa substância ${ }^{4}$.

Existem diversas terminologias para conceituar os padrões de consumo do álcool. Nesse trabalho optou-se pelas definições adotadas pela Organização Mundial da Saúde (OMS), a saber: 1) Consumo moderado ou de baixo risco: quando as doses ingeridas são moderadas e não causam problemas; 2) Beber problemático ou abusivo: quando o consumo de

\footnotetext{
Assistente social. Mestre, Programa de Pós-Graduação em Saúde Ambiental e Saúde do Trabalhador, Instituto de Geografia, Universidade Federal de Uberlândia. Cirurgião-dentista. Mestre, Programa de Pós-Graduação em Odontologia, Faculdade de Odontologia, Universidade Federal de Minas Gerais, Belo Horizonte, Minas Gerais, Brasil.

Cirurgiã-dentista. Doutora, Departamento de Odontologia Preventiva e Social, Universidade Federal de Uberlândia, Uberlândia, Minas Gerais, Brasil.
} 
bebidas causa problemas de ordem individuais ou coletiva, de saúde ou sociais; 3 ) Consumo compulsivo periódico de bebida (em inglês: binge drinking), quando ocorre ingestão intensa durante um período prolongado, escolhido de maneira propositada ${ }^{5}$.

A identificação do padrão de consumo de álcool entre os trabalhadores nas diferentes realidades de trabalho corresponde à estratégia inicial de promoção da saúde e à prevenção de agravos decorrentes do consumo abusivo de álcool, tanto para o trabalhador, quanto para a família e a sociedade ${ }^{6}$. Dessa forma, o objetivo deste estudo foi identificar o padrão e a prevalência de consumo de bebidas alcoólicas entre os trabalhadores de equipes de Saúde da Família.

\section{Materiais e método}

Trata-se de um estudo transversal realizado com trabalhadores de equipes de Saúde da Família do município de Uberlândia, interior de Minas Gerais, considerado o segundo município mais populoso do Estado (669.672 habitantes). A pesquisa foi submetida à avaliação ética e aprovada pelo Comitê de Ética em Pesquisa com Seres Humanos da Universidade Federal de Uberlândia (CAAE 50469015.3.0000.5152).

A cobertura de equipes de saúde da família do município no momento da pesquisa era de $39,84 \%$, alcançados em um universo de 74 equipes de saúde da família, 27 equipes de saúde bucal e 10 equipes do Núcleo de Apoio à Saúde da Família, totalizando 967 trabalhadores de saúde da família. A coleta de dados ocorreu entre fevereiro e junho de 2016.

Foram incluídos no estudo os trabalhadores das equipes mínimas de Saúde da Família (médico, enfermeiro, técnicos ou auxiliares de enfermagem e agentes comunitários de saúde), de Saúde Bucal da Família (cirurgião-dentista, auxiliar e técnico de saúde bucal), trabalhadores da equipe de apoio (auxiliar de serviços gerais, oficiais e auxiliares administrativos e segurança patrimonial) e profissionais do Núcleo de Apoio a Saúde da Família (assistente social, psicólogo, nutricionista, fisioterapeuta, educador físico e farmacêutico).

Para o cálculo amostral, considerou-se uma precisão de $8 \%$ e um nível de confiança de $95 \%$, resultando em uma amostra de 130 trabalhadores de saúde. Para seleção desses trabalhadores, foram identificadas as oito equipes de saúde da família com maior tempo de funcionamento em cada um dos seis setores sanitários do município, de acordo com a data de inserção no Cadastro Nacional de Estabelecimentos de Saúde. Nos setores em que foram identificadas mais de uma unidade de saúde cadastradas na mesma data, procedeu-se o sorteio da equipe para inclusão no estudo. Os questionários foram distribuídos aos 130 profissionais, mas somente $90,77 \%(n=118)$ foram considerados apropriados para a análise de dados. Optou-se por fazer a seleção a partir das equipes de saúde e não por ca- tegorias profissionais por considerar que o conjunto de profissionais que formam uma equipe de saúde estão sujeitos às mesmas complexidades para o trabalho em um determinado território.

Foram utilizados dois instrumentos de autopreenchimento: o perfil sociodemográfico e ocupacional, desenvolvido pelos autores a partir do modelo utilizado pelo Instituto Brasileiro de Geografia e Estatística; e o teste de identificação dos transtornos do uso de álcool (Audit), instrumento criado pela OMS e validado no Brasil por Méndez ${ }^{7}$ para identificar níveis de consumo de álcool.

Perfil sociodemográfico e ocupacional: foram coletadas as variáveis categóricas ordinais: faixa etária em anos (18 - 30, 31 a 40, 41 a 60$)$, escolaridade (fundamental, médio, superior, especialização, mestrado), renda familiar em salários (até 2 , entre 2 e 5 , entre 6 e 10, e mais que 10), tempo de trabalho em anos (menor que 1 , entre 1 e 5 , entre 6 e 10, maior que 10) e categoria profissional (equipe de apoio, agente comunitário de saúde, profissionais de nível técnico e profissionais de nível superior); como variáveis categóricas nominais: sexo (masculino, feminino), estado civil (solteiro, casado, divorciado, viúvo) e vínculo (celetista, celetista e estatutário, estatutário); como variável numérica restrita: o número de vínculos empregatícios. É indispensável destacar que o Audit é um questionário de rastreamento e não de diagnóstico.

Esse instrumento é composto por dez questões e avalia o uso recente de álcool, sintomas de dependência e problemas relacionados ao álcool. As respostas a cada questão são pontuadas de 1 a 4 , sendo as maiores pontuações indicativas de problemas. Esse é um instrumento de fácil e rápida aplicação, necessitando apenas de um treinamento simples para a codificação dos dados. Os escores do Audit geram variáveis numéricas que variam de 0 a 40, as quais podem ser agrupadas em quatro variáveis categóricas ordinais: zona I - os escores de 0 a 7 identificam consumo de baixo risco ou abstêmios; zona II - os escores entre 8 e 15 apontam um consumo de risco; zona III - os escores entre 16 e 19 fazem referência ao uso nocivo ou consumo de alto risco; zona IV - os escores entre 20 a 40 indicam uma provável dependência.

As variáveis em estudo foram analisadas de forma descritiva com o auxílio do software SPSS versão 20.1.

\section{Resultados}

Foram analisados os questionários de 118 , sendo 64 das equipes mínimas de saúde da família, 12 das equipes saúde bucal da família, 21 trabalhadores da equipe de apoio e 21 profissionais do Núcleo de Apoio à Saúde da Família. Especificamente, participaram do estudo, 7 Assistentes Sociais, 41 Agente Comunitário de Saúde, 4 Agentes Patrimoniais, 1 Agente de Controle de Zoonoses, 7 Auxiliares de 
Serviços Gerais, 3 Auxiliares Saúde Bucal, 6 Cirurgiões-Dentistas, 1 Educador Físico, 6 Enfermeiros, 1 Farmacêutico, 3 Fisioterapeutas, 9 Médicos, 4 Nutricionistas, 9 Oficiais Administrativos, 5 Psicólogos, 8 Técnicos de Enfermagem e 3 Técnicos de Saúde Bucal.

A caracterização sociodemográfica e ocupacional dos profissionais está descrita na Tabela 1. Observou-se que os participantes do estudo eram principalmente do sexo feminino ( $\mathrm{n}=102 ; 86,41 \%)$, casados $(n=56 ; 47,5 \%)$ e na faixa etária entre 41 e 60 anos $(\mathrm{n}=48 ; 40,7 \%)$. Prevaleceram os trabalhadores que se disseram participantes da religião católica $(\mathrm{n}=; 52,5 \%)$, com renda familiar entre três e cinco salários mínimos ( $\mathrm{n}=42 ; 35,6 \%)$. Os agentes comunitários de saúde compuseram a principal catego- ria profissional do estudo $(\mathrm{n}=39 ; 34,7 \%)$. Verificou-se, ainda, que a maioria dos entrevistados tinham apenas um vínculo empregatício $(\mathrm{n}=111 ; 94,1 \%)$ e trabalhavam na saúde da família entre um e cinco $\operatorname{anos}(\mathrm{n}=60 ; 50,8 \%)$.

A Tabela 2 apresenta o padrão de consumo de álcool pelos participantes e os problemas decorrentes. Essas perguntas foram direcionadas apenas àqueles que afirmaram fazer uso de álcool $(\mathrm{n}=59$; $50 \%$ ). A quantidade de doses mais frequente foi de 1 a 2 doses e $2 \%$ consumiam álcool quase todos os dias, $41 \%$ revelaram ter consumido seis ou mais doses de álcool em uma única ocasião (binge). No que diz respeito à frequência dos episódios de binge, 19\% alegaram que o episódio ocorre pelo menos uma vez por mês.

Tabela 1 - Distribuição das variáveis sociodemográficas e ocupacionais dos trabalhadores das equipes de saúde da família do município de Uberlândia, MG, 2016 ( $n=118)$

\begin{tabular}{|c|c|c|c|}
\hline & \\
\hline & Variáveis & $\mathrm{N}$ & $\%$ \\
\hline \multirow{2}{*}{ Sexo } & Feminino & 102 & 86,4 \\
\hline & Masculino & 16 & 13,6 \\
\hline \multirow{4}{*}{ Faixa etária } & 18 a 30 anos & 28 & 23,7 \\
\hline & 31 a 40 anos & 42 & 35,6 \\
\hline & 41 a 60 anos & 48 & 40,7 \\
\hline & Mais de 61 anos & 0 & 0,0 \\
\hline \multirow{5}{*}{ Estado civil } & Casado & 56 & 47,5 \\
\hline & Solteiro & 32 & 27,1 \\
\hline & União estável & 14 & 11,9 \\
\hline & Divorciado & 11 & 9,3 \\
\hline & Outros & 5 & 4,2 \\
\hline \multirow{5}{*}{ Religião } & Católicos & 62 & 52,5 \\
\hline & Protestantes & 30 & 25,4 \\
\hline & Sem religião & 14 & 11,9 \\
\hline & Kardecistas & 8 & 6,8 \\
\hline & Outras religiões & 4 & 3,4 \\
\hline \multirow{5}{*}{ Renda mensal familiar } & Até 2 salários mínimos & 31 & 26,3 \\
\hline & De 3 a 5 salários mínimos & 42 & 35,6 \\
\hline & De 6 a 10 salários mínimos & 15 & 12,7 \\
\hline & Mais de 10 salários mínimos & 16 & 13,6 \\
\hline & Prefiro não declarar & 14 & 11,9 \\
\hline \multirow{3}{*}{ Escolaridade } & Ensino fundamental & 9 & 7,6 \\
\hline & Ensino médio & 64 & 54,2 \\
\hline & Ensino superior & 45 & 38,1 \\
\hline \multirow{4}{*}{$\begin{array}{l}\text { Tempo de trabalho na Saúde } \\
\text { da Família }\end{array}$} & $<1$ ano & 20 & 16,9 \\
\hline & 1 a 5 anos & 60 & 50,8 \\
\hline & 5 a 10 anos & 22 & 18,6 \\
\hline & $>10$ anos & 16 & 13,6 \\
\hline Número de vínculos & 1 & 111 & 94,1 \\
\hline empregatícios & 2 & 7 & 5,9 \\
\hline
\end{tabular}

Fonte: autores. 
Tabela 2 - Distribuição de quantidade e frequência dos sintomas e problemas associados ao consumo de álcool entre os profissionais das equipes de saúde da família, Uberlândia, MG, $2016(n=59)$

\begin{tabular}{|c|c|c|c|}
\hline Item & Descritivo & $\mathrm{N}$ & $\%$ \\
\hline \multirow{5}{*}{$\begin{array}{l}\text { Número de doses consumidas } \\
\text { em dia típico }\end{array}$} & $1-2$ & 30 & 51 \\
\hline & $3-4$ & 20 & 34 \\
\hline & $5-6$ & 6 & 10 \\
\hline & $7-8$ & 0 & 0 \\
\hline & 9 ou mais & 3 & 5 \\
\hline \multirow{5}{*}{$\begin{array}{l}\text { Frequência de cinco ou mais } \\
\text { doses em uma única ocasião }\end{array}$} & Nunca & 35 & 59 \\
\hline & Menos que uma vez ao mês & 11 & 19 \\
\hline & Mensalmente & 7 & 12 \\
\hline & Semanalmente & 5 & 8 \\
\hline & Todos ou quase todos os dias & 1 & 2 \\
\hline \multirow{4}{*}{$\begin{array}{c}\text { Frequência de beber sem } \\
\text { conseguir parar após ter } \\
\text { começado }\end{array}$} & Nunca & 54 & 91 \\
\hline & Menos que uma vez ao mês & 3 & 5 \\
\hline & Mensalmente & 1 & 2 \\
\hline & Todos ou quase todos os dias & 1 & 2 \\
\hline \multirow{5}{*}{$\begin{array}{c}\text { Deixou de fazer algo } \\
\text { esperado ou de responder um } \\
\text { compromisso devido ao uso de } \\
\text { álcool }\end{array}$} & Nunca & 55 & 93 \\
\hline & Menos que uma vez ao mês & 4 & 7 \\
\hline & Mensalmente & 0 & 0 \\
\hline & Semanalmente & 0 & 0 \\
\hline & Todos ou quase todos os dias & 0 & 0 \\
\hline \multirow{5}{*}{$\begin{array}{l}\text { Necessidade de beber uma } \\
\text { primeira dose pela manhã para } \\
\text { sentir-se melhor }\end{array}$} & Nunca & 58 & 98 \\
\hline & Menos que uma vez ao mês & 1 & 2 \\
\hline & Mensalmente & 0 & 0 \\
\hline & Semanalmente & 0 & 0 \\
\hline & Todos ou quase todos os dias & 0 & 0 \\
\hline \multirow{5}{*}{$\begin{array}{c}\text { Frequência de sentimento de } \\
\text { culpa ou remorso depois de } \\
\text { beber }\end{array}$} & Nunca & 51 & 86 \\
\hline & Menos que uma vez ao mês & 6 & 10 \\
\hline & Mensalmente & 1 & 2 \\
\hline & Semanalmente & 1 & 2 \\
\hline & Todos ou quase todos os dias & 0 & 0 \\
\hline \multirow{5}{*}{$\begin{array}{c}\text { Frequência de incapacidade em } \\
\text { lembrar-se do que aconteceu } \\
\text { na noite anterior porque havia } \\
\text { bebido }\end{array}$} & Nunca & 54 & 92 \\
\hline & Menos que uma vez ao mês & 4 & 7 \\
\hline & Mensalmente & 1 & 2 \\
\hline & Semanalmente & 0 & 0 \\
\hline & Todos ou quase todos os dias & 0 & 0 \\
\hline
\end{tabular}

Fonte: autores.

Quanto aos sintomas e problemas relacionados ao álcool, nota-se que 9\% dos entrevistados apresentaram a percepção de não obter êxito em parar de beber uma vez que tinham iniciado (menos que uma vez ao mês, mensalmente, ou todos ou quase todos os dias); que 7\% já tinham deixado de fazer o que era esperado devido ao consumo de álcool; e que apenas $2 \%$ assumiram necessidade de beber pela manhã para se sentir bem ao longo do dia. Verificou-se ainda que $86 \%$ dos trabalhadores nunca sentiram culpa ou remorso depois de beber enquanto 9\% tiveram apagamento.
Com base nas últimas questões do Audit, que se referem aos problemas relacionados ao uso de álcool e foram aplicadas aos 118 participantes do estudo, observou-se que $3 \%$ alegaram ter causado ferimentos ou prejuízos a si mesmo ou a outras pessoas após ingestão de álcool e que 6\% foram aconselhados a parar de beber.

A Tabela 3 apresenta a distribuição dos trabalhadores nas chamadas zonas de risco do Audit. A maioria dos entrevistados $(n=109 ; 92 \%)$ da amostra foram classificados como usuários compatível com baixo risco, $6 \%(n=7)$ dos entrevistados foram identificados como consumidores de uso de risco. 
Tabela 3 - Caracterização do consumo de álcool de acordo com as zonas de risco pelos trabalhadores das equipes saúde da família do município de Uberlândia, MG, 2016 (n=118)

\begin{tabular}{l|c|c|c}
\hline \multicolumn{1}{c|}{ Níveis de risco } & Escores & N & $\%$ \\
\hline Zona I (baixo risco) & 0 a 7 & 109 & 92 \\
Zona II (uso de risco) & 8 a 15 & 7 & 6 \\
Zona III (uso nocivo) & 16 a 19 & 1 & 1 \\
Zona IV (provável dependência) & 20 a 40 & 1 & 1 \\
\hline
\end{tabular}

Fonte: autores.

\section{Discussão}

O estudo possibilitou conhecer o perfil socioeconômico e ocupacional dos trabalhadores da saúde da família, bem como identificar o padrão de consumo, sintomas de dependência e os problemas relacionados ao uso de álcool. Verificou-se que, enquanto a metade dos trabalhadores referiram não fazerem consumo de bebida alcoólica, a outra metade declarou uso em alguma frequência nos últimos doze meses. Esse resultado está alinhado com uma pesquisa realizada pelo II Levantamento Nacional de Álcool e Drogas ${ }^{8}$, no qual foi estimado que a metade da população brasileira é abstêmia, $32 \%$ exibem prática de beber moderado e $16 \%$ consomem quantidades nocivas de álcool. A prevalência dos profissionais que apresentaram um padrão de consumo apontado como excessivo, denotando riscos à saúde, foi de $8 \%$ (Zonas II, III e IV do Audit), dado inferior ao observado pelo levantamento anteriormente citado, em que se observou um uso nocivo igual a $16 \% 8$.

Um estudo realizado por Rocha e Davis ${ }^{9}$, com o objetivo de descrever e discutir a prevalência e o padrão de consumo de álcool e outras drogas entre profissionais de saúde demonstrou que em torno de $67,6 \%$ concordaram que o uso de drogas por funcionários pode estar relacionado ao estresse e/ou a condições de trabalho.

Pesquisa realizada por Oliveira et al. ${ }^{10}$, para descrever os padrões de uso de álcool por trabalhadores de enfermagem e a associação com o trabalho, revelou que 26,6\% dos trabalhadores associaram o consumo de bebidas alcoólicas com o trabalho e $18,8 \%$ dos entrevistados referiram insatisfações e estresse decorrentes do trabalho e 9,9\% identificaram prejuízos laborais como: absenteísmo, atrasos, trabalhar sobre a influência de álcool e conflitos com a autoridade no trabalho.

Os resultados do padrão binge de consumo, considerado de risco, foi observado em $41 \%$ dos trabalhadores que fizeram consumo de bebida alcoólica nos últimos 12 meses. Importante ressaltar que os episódios de binge estão ligados a problemas importantes com comportamentos mais arriscados, por exemplo, sexo desprotegido (gravidez indesejada e doenças sexualmente transmissíveis), violências de todos os tipos, agravos à saúde, aumento da criminalidade e evasão escolar, entre outros inúmeros riscos. Estudo sobre o perfil dos indivíduos que bebem nesse padrão, realizado no Brasil por Castroand et al. ${ }^{11}$, indicou que os adultos têm maior possibilidade de padecer com as consequências desse uso.

O consumo de bebidas alcoólicas encontrados nesse estudo foi confrontado com o consumo da população brasileira em geral em que $28 \%$ dos adultos bebem em classificação binge, $24 \%$ bebem sem essa classificação (binge), 3\% fazem uso nocivo de álcool e $9 \%$ são considerados dependentes ${ }^{11}$.

Compreendendo que o consumo de álcool identificado pelos trabalhadores pode ser inerente à história cultural presente na sociedade desde os tempos mais primitivos e é visto como complemento dos momentos de alegria e de festa, no quais as pessoas se reúnem para comemorar e celebrar a vida ${ }^{12}$. As primeiras informações sobre o uso do álcool são de 6.000 a.C., muito utilizado por seus efeitos desinibidor e antidepressivo, bem como pelo fácil acesso, tornando-se um dos problemas de saúde que afetam homens e mulheres em qualquer idade e em todas as classes sociais ${ }^{13}$.

Mesmo o consumo de álcool tendo assumido uma característica de normalidade na cultura popular, durante anos, o alcoolismo foi considerado como um problema moral, fruto da falta de caráter da pessoa, sendo que, posteriormente, passou a ser considerado uma doença, cujo um dos pressupostos é que os dependentes teriam características genéticas e de personalidade diferentes do restante da população $0^{14}$.

Posto isso, para o enfrentamento do consumo de álcool, torna-se importante analisar a prevalência e os determinantes dos hábitos de riscos, levando em consideração que são vários fatores agregados e não apenas as inclinações pessoal e psíquica dos indivíduos que determinam os componentes etiológicos multicausais para o agravamento do uso de álcool. $\mathrm{Na}$ investigação de trabalhos bibliográficos com ênfase na compreensão etiológica do alcoolismo realizada por Moraes e Barrocco ${ }^{15}$, foram destacados os determinantes biológicos orgânicos, psicológicos e comportamentais, sociais e ambientais.

No trabalho de $\mathrm{Lima}^{3}$, que retrata o aprofundamento no envolvimento de como acontece a relação entre trabalho e uso de álcool, apresentou comprovações importantes que em múltiplos contextos profissionais surge o desenvolvimento de quadros de consumo de álcool. Os estudos foram realizados em torno de trabalhadores da construção civil, policiais militares, cobradores e motoristas de ônibus urbano, categorias identificadas por estudos epidemiológicos com problemas relacionados ao uso de álcool. Além dessas também são relacionadas profissões como: garis, bombeiros, mecânicos de veículos e advogados.

Para a autora, o uso de álcool se tornou uma maneira de enfrentar situações adversas e inerentes às atividades profissionais, dentre as quais destacam-se as frustrações, as pressões, os ritmos acelerados, a competitividade, os desgastes físicos, as relações desiguais, a falta de autonomia, os conflitos com a gerência, a falta de clareza nas regras e outros fatores ${ }^{3}$. 


\section{Considerações finais}

Este estudo, de caráter exploratório, possibilitou a construção de conhecimento sobre os padrões de consumo de álcool dos trabalhadores da saúde da família, um campo que tem empregado, progressivamente, mais profissionais de saúde. Observou-se que o uso de álcool nos últimos doze meses por esses trabalhadores foi semelhante ao observado pela população em geral. Contudo, entre os que fazem uso do álcool, foi observada uma porcentagem importante de trabalhadores que fazem consumo em binge.

Estudos como este são relevantes para orientar ações de prevenção ao uso prejudicial do álcool. Além do mais, são indicativos e subsidiam os gestores do Serviço de Saúde do Trabalhador, tendo em vista a escassez de pesquisas de rastreamento do padrão de consumo de álcool entre trabalhadores da saúde, em especial nesse momento da saúde da família.

Vale ressaltar, que não se pretende afirmar que todo padrão de uso de álcool esteja relacionado unicamente ao trabalho, porém se destaca a importância de reflexões a respeito das condições e da organização do trabalho como fatores colaboradores para a manifestação.

\section{Abstract}

Objective: to identify the pattern and prevalence of alcoholic beverage consumption among workers of Family Health Teams. Method: cross-sectional study performed with 118 family health workers. The data were collected by means of a socioeconomic and occupational questionnaire and the Alcohol Use Disorders Identification Test, which results were analyzed descriptively. Results: alcohol consumption was observed in $50 \%$ of health professionals. From those who consumed alcohol, $41 \%$ reported having consumed six or more doses of alcohol on a single occasion (binge), $19 \%$ reported at least one binge episode per month, $12 \%$ reported it monthly, $8 \%$ weekly, and $2 \%$ daily or almost every day. Conclusions: the alcohol consumption among workers of Family Health Teams was considered similar to the Brazilian population. However, among the workers who consume alcohol, the binge frequency stood out.

Keywords: Alcoholism. Primary health care. Occupational health.

\section{Referências}

1. Brasil. Política Nacional de Atenção Básica. Ministério da Saúde. Secretaria de Atenção à Saúde. Departamento de Atenção Básica. Brasília, DF: Ministério da Saúde; 2012.

2. Le Guillant L. Escritos de Louis de Le Guillant: da ergoterapia à psicopatologia do trabalho [Tradução de Guilherme Teixeira]. Petrópolis: Vozes; 2006.

3. Lima MEA. Álcool e trabalho: revisitando conceitos à luz de novas descobertas. Curitiba: Juruá; 2015.

4. Vaissman M. Alcoolismo no trabalho. Rio de Janeiro: Fiocruz; 2004.

5. Organização Mundial de Saúde. Self-help strategies for cutting down or stopping substance use: a guide. Genebra: OMS; 2010.

6. Farias LO, Vaitsman J. Interação e conflito entre categorias profissionais em organizações hospitalares públicas. Cad Saúde Pública 2002; 18(5):1229-41.

7. Méndez EB. Uma versão brasileira do AUDIT- Alcohol Use Disorders Identification Test [Dissertação de Mestrado]. Pelotas: Universidade Federal de Pelotas, 1999.

8. Laranjeiras R. II Levantamento Nacional de Álcool e Drogas. São Paulo: Unifesp; 2014.

9. Rocha PR, David HMSL. Padrão de consumo de álcool e outras drogas entre profissionais de saúde: retrato de alunos de cursos lato sensu de uma instituição pública. SMAD 2015; 11(1):42-8.

10. Oliveira EB, Fabri JMG, Paula GS, Souza SRC, Silveira WG, Matos GS. Padrões de uso de álcool por trabalhadores de enfermagem e a associação com o trabalho. Rev Enferm UERJ 2013; 21(6):729-35.

11. Castroand DS, Sanchez ZM, Zaleski M, Alves HN, Pinsky I, Caetano R, et al. Sociodemographic Characteristis associated with binge drinking amang Brazilians. Drug Alcohol Dependence 2012; 126(1):272-6.

12. Gigliotti A, Bessa MA. Síndrome de dependência do álcool: critérios diagnósticos. Rev Bras Psiquiatr 2004; 26(supl. 1):11-3.

13. Mansur J. O que é alcoolismo. São Paulo: Brasiliense; 2004.

14. Almeida MGB. A violência na sociedade contemporânea. Porto Alegre: EDIPUCRS; 2010.

15. Moraes RJS, Barroco SMS. Concepções do alcoolismo na atualidade: pesquisas hegemônicas, avanços e contradições. Rev Bras Psi Teor Pesq 2016; 32(1):229-37.

\section{Endereço para correspondência:}

Liliane Parreira Tannús Gontijo

Av. Pará 1720, Bloco 2G, Sala 01

Campus Umuarama, Bairro Umuarama

38405320, Uberlândia, MG, Brasil

Telefone: (34) 3225-8145

E-mail: lilianetannus1@gmail.com

Recebido: 16/07 / 18. Aceito: 19/07/ 18. 